11.10.Kk., 04.50.-h.

To appear in Phys. Rev. D, 2010

\title{
Exact solutions for a Maxwell - Kalb-Ramond action with dilaton: localization of massless and massive modes in a sine-Gordon brane-world
}

\author{
H. R. Christiansen, M. S. Cunha, M. O. Tahim \\ Grupo de Física Teórica, State University of Ceara (UECE) - Av. Paranjana, \\ 1700, CEP 60740-903, Fortaleza, Ceará, Brazil
}

\begin{abstract}
We analytically find the exact propagation modes of the electromagnetic and the Kalb-Ramond fields together in a five-dimensional curved space-time. The existence and localization of gauge particles into our four-dimensional world (4D) is studied in detail on a brane-world scenario in which two gauge fields interact with a dilaton and a gravitational background. The coupling to the dilaton is different in each case causing the splitting between both gauge spectra. The gauge field zero-modes and an infinite tower of Kaluza-Klein massive states are analytically obtained. Relevant conditions on the dilaton coupling constant are found in order to identify with precision every finite tensor and vector eigenstate in the theory. An exact quantization condition on the whole mass spectrum, depending on the dilaton coupling constant and the bulk Planck mass, is inherited from the extra-dimension. This allows finding an exact rule to prevent tachyons in the theory and, by the same token, predicting a possible Kalb-Ramond tensor zero-mode in 4D world. We also show that KK massive-modes contributions onto 4D physics are strongly suppressed.
\end{abstract}

PACS numbers:

Keywords: Extra-dimensions, Localization, Kalb-Ramond, Dilaton, Kaluza-Klein, Sine-Gordon 


\section{INTRODUCTION}

Together with the mass question, one of the most difficult issues to understand in the Standard Model is how the electroweak scale can be perturbatively stable at seventeen orders of magnitude below the Planck scale. In other words, whether there is a way to bring the quantum gravity scale below $\sim 1 \mathrm{TeV}$.

Recent proposals involving high-dimensional models and brane-world scenarios raise possible solutions to such a hierarchy problem of gauge couplings [1]. Ten-dimensional superstring theory allows these kind of frameworks to get embedded within a theory of everything. Since extra-dimensional theories present more degrees of freedom than four-dimensional (4D) ones, they provide a richer framework to approach physical phenomena. The problem is that the parameter space to be covered is much wider and calculations in higher dimensional gravity are technically very difficult.

The main goal of high-dimensional theories being the inclusion of gravity together with the Standard Model interactions introduces nontrivial changes in all sectors, so gauge forces have to be proven to exist as needed in the ordinary space slice. In the gauge sector the analysis of localization becomes crucial to have a $4 \mathrm{D}$ effective result with massless photons. Modeling our universe as a domain wall has been motivated by the D-brane solutions of string theory. Gauge fields are deposited on D-branes from open strings ending on them. However, domain walls can be constructed in field theoretic frameworks with no direct possibility to localize gauge fields. As a matter of fact, in 5D we shall need not only gravitational fields but also a dilaton field to properly accomplish this task [2, 3]. Since simple domain walls cannot hold gauge fields in [4], this scalar will show to be essential to guarantee the existence of $\mathrm{U}(1)$ gauge fields localized in the four-dimensional world.

Current interest in theories with bosonic fields of different spin arise as a result of their existence on conformally flat spaces of any dimensionality $(D \geq 4)$ such as anti-de Sitter geometries. The role of tensor bosons in the context of AdS/CFT correspondence, is particularly important when coupled to gravity [5]. String theory is so far mainly based on its low spin excitations and their low energy interactions. The low energy 4D effective field theory action of string theory [6] contains two massless fields: a second rank antisymmetric tensor from the (Neveu-Schwarz) NS-NS sector of the underlying string theory known as Kalb-Ramond field [7] and a scalar field called dilaton. The Kalb-Ramond field, provided 
the lagrangian is invariant under a second gauge transformation, has three free components. However, it is known that classically a free antisymmetric tensor field is dual to a scalar which involves just one degree of freedom. Indeed, the third rank field-strength corresponding to the the Kalb-Ramond field is dual to a pseudo-scalar in 4D so-called axion. Consequences of the presence of the axion in a curved space-time on some physical phenomena have been investigated in view of possible indirect evidences of string theory at low energies [8].

Here, we will focus on both Maxwell and Kalb-Ramond gauge fields together, in a warped five-dimensional bulk with a dilaton and a brane defect that mimics the ordinary world. This thick brane configuration results from a field action with a (deformed) sine-Gordon potential that depends on two scalars. One of these is a kink representing the membrane itself and the other the dilaton in a field theoretic scenario. Metric, dilaton and brane configurations are geometrically consistent solutions of the world action. On such an interesting background we shall analytically obtain all the eigenstates for both gauge fields, viz. zero-modes, massive modes and tachyons building both whole spectra.

Regarding the propagating modes of the Kalb-Ramond field, so far only simple and qualitative calculations have been obtained in this context. In the present paper, we perform a systematic search by means of an analytical approach. Furthermore, we also include the electromagnetic field together, in order to exhibit their actual differences. We thus show that vector and tensor spectra are analogous but not equal as a consequence of their different coupling to the dilaton. In both cases, the full tower of gauge massive modes, so far invisible in ordinary world, are proven to be strongly suppressed in 4D. We also predict that a localized zero-mode is not only possible for the 5D electromagnetic field but also, and simultaneously, for a 5D Kalb-Ramond field, provided the dilaton coupling constant is above a certain value. This value is precisely that needed to exclude tachyons in the theory. All of this amounts to show that the model presented here is an interesting arena to discuss extra-dimensional physics and that our ordinary 4D world seems to be compatible with a higher dimensional universe, apparently of a 4D stringy brane nature.

The paper is organized as follows. In the next Section, we present the geometrical background. Along with a conveniently warped metric, this includes a sine-Gordon potential depending on the brane and the dilaton fields. In previous treatments a Higgs like potential has been preferred, so we shall briefly address this case as a warm up. In Section III we introduce the action for the gauge fields in the bulk, Maxwell and Kalb-Ramond, coupled 
to a warped gravity and dilaton background. We next derive the bulk equations of motion and separate the extra coordinate from the four-dimensional world coordinates. Then, in Section [V], we find a condition for localization and transform the equations of motion for the gauge fields into a couple of Schrodinger like equations. In the following sections we analytically discuss the Maxwell and Kalb-Ramond spectra as a function of the dilaton coupling constant. We particularly emphasize the Sturm-Liouville nature of the differential equations resulting from the mapping of the original problem, and show significant consequences on the space of solutions. In Section $\square$ we obtain exact expressions for the full spectrum of eigenstates of the problem. Analyticity constraints on massive modes, zero-modes and tachyons are discussed. Kaluza-Klein eigenstates are fully exhibited and we show that massive modes are strongly suppressed on the brane so that ordinary four-dimensional gauge interactions are not significantly modified in the present set up. Finally, in Section VI we draw our conclusions.

\section{THE SPACE-TIME BACKGROUND: GRAVITATIONAL WARPING AND DILATON}

We start our analysis by studying the appropriate space-time framework for the description of a consistent gauge theory. In this Section we will show how one proceeds with both vector and tensor gauge fields in order to look for zero-modes that can be localized in a four-dimensional membrane embedded in a five-dimensional space-time. Here, the extra dimension is not necessarily an orbifold and will be assumed infinite. Furthermore, the brane is not included in the model as a static external source but it is dynamically obtained as a solution to the Einstein equations for gravity coupled to (two) scalar fields. One of these scalars is for creating a domain wall defect (a thick brane) while the other is the dilaton field.

It is worth noting that either gravitational and fermionic massless quanta can be trapped in four-dimensional (4D) domain walls lying within a five-dimensional (5D) bulk with some AdS like metric [2, 9]. On the other hand, it is known that vector gauge bosons in these kind of scenarios are not localizable unless the coupling constant is dynamically modified. The reason is that gauge field theory is conformal [10] so that all the information coming from the metric warping factors automatically drops out resulting in a non-normalizable 
zero-mode. Fortunately, the coupling of the dilaton to the gauge field in the kinetic term modifies the rescaling properties allowing for the localization of the vector zero-mode [2] and tensor zero-mode [11] respectively (see also [12]).

Following this approach and motivated by low-energy string theories, here we will discuss both Maxwell and Kalb-Ramond gauge fields together, coupled to a gravitational background and a consistent dilaton configuration. In what follows, we shall obtain close expressions for the gauge modes in the 5D space-time. This will be in order to analytically discuss the phenomenological consequences, not only of zero-modes but also of massive states.

First, it is necessary to obtain a solution to the equations of motion of the gravitational field for a potential functional depending on both the dilaton and membrane field variables [2]. We therefore analyze the following action for two real scalar fields

$$
S_{B}=\int d^{4} x d y \sqrt{-\operatorname{det} G_{M N}}\left[2 M^{3} R-\frac{1}{2}(\partial \Phi)^{2}-\frac{1}{2}(\partial \Pi)^{2}-\mathcal{V}(\Phi, \Pi)\right]
$$

where $M$ is the Planck constant in $5 \mathrm{D}$, and $R$ is the Ricci scalar. The solution for $\Phi$ is the membrane kinking on our 4D-world. The corresponding field solution for $\Pi$ will be the dilaton configuration consistent with the metric and the kink. As usual we adopt latin capitals on the bulk and greek lower case letters on 4D.

We next shall assume some ansatz for the space-time metric

$$
d s^{2}=e^{2 \Lambda(y)} \eta_{\mu \nu} d x^{\mu} d x^{\nu}+e^{2 \Sigma(y)} d y^{2}
$$

where $\Lambda$ and $\Sigma$ are warp functions that depend just on the extra (fifth) coordinate, and $\operatorname{diag}(\eta)=(-1,1,1,1)$. The equations of motion for action (1) are

$$
\begin{aligned}
& \frac{1}{2}\left(\Phi^{\prime}\right)^{2}+\frac{1}{2}\left(\Pi^{\prime}\right)^{2}-e^{2 \Sigma(y)} \mathcal{V}(\Phi, \Pi)=24 M^{3}\left(\Lambda^{\prime}\right)^{2}, \\
& \frac{1}{2}\left(\Phi^{\prime}\right)^{2}+\frac{1}{2}\left(\Pi^{\prime}\right)^{2}+e^{2 \Sigma(y)} \mathcal{V}(\Phi, \Pi)=-12 M^{3} \Lambda^{\prime \prime}-24 M^{3}\left(\Lambda^{\prime}\right)^{2}+12 M^{3} \Lambda^{\prime} \Sigma^{\prime},
\end{aligned}
$$

and

$$
\begin{aligned}
& \Phi^{\prime \prime}+\left(4 \Lambda^{\prime}-\Sigma^{\prime}\right) \Phi^{\prime}=e^{2 \Sigma} \frac{\partial \mathcal{V}}{\partial \Phi} \\
& \Pi^{\prime \prime}+\left(4 \Lambda^{\prime}-\Sigma^{\prime}\right) \Pi^{\prime}=e^{2 \Sigma} \frac{\partial \mathcal{V}}{\partial \Pi}
\end{aligned}
$$

where the prime means derivative with respect to $x^{5}=y$ (note the correction in eq. (44) with respect to Ref.[2]). 
In order to solve this system we use a supergravity motivated [13] potential functional $\mathcal{W}(\Phi)$. This so-called superpotential is applicable to non-supersymmetric domain walls [14] as the present one, and is defined by

$$
\Phi^{\prime}=\frac{d \mathcal{W}}{d \Phi}
$$

In the absence of gravity, for a double-well potential of the Higgs type $V(\Phi)=\frac{\delta}{4}\left(\Phi^{2}-v^{2}\right)^{2}$, the simplest possible static membrane configuration dependent on the fifth coordinate is a bounce

$$
\Phi(y)=v \tanh (\kappa y)
$$

[2], consistent with the superpotential

$$
\mathcal{W}(\Phi)=v \kappa \Phi\left(1-\frac{\Phi^{2}}{3 v^{2}}\right)
$$

where $\kappa^{2}=\delta v^{2} / 2$.

Putting into scene the metric ansatz (2), the dilaton field $\Pi(y)$, and taking into account the equations of motion, the necessary potential consistent with the bounce can be written as

$$
\mathcal{V}(\Phi, \Pi)=\exp \left(\Pi / \sqrt{12 M^{3}}\right) \cdot\left(\frac{1}{2}\left(\frac{d \mathcal{W}}{d \Phi}\right)^{2}-\frac{5}{32 M^{3}} \mathcal{W}(\Phi)^{2}\right)
$$

Now, one obtains the following solution to the equations of motion, eq.(3) and eq.(4), consistent with the bounce configuration eq.(6) and the potential eq.(8)

$$
\Lambda(y)=4 \Sigma(y)=\frac{-\Pi(y)}{\sqrt{3 M^{3}}}=-\beta\left(\ln \cosh ^{2}(\kappa y)+\frac{1}{2} \tanh ^{2}(\kappa y)\right),
$$

where $\beta=v^{2} / 36 M^{3}$.

The possibility of considering a stack of traveling branes of this kind, or even colliding branes, can be addressed by means of a sine-Gordon potential, which in the absence of gravity is very well-known and has the form

$$
V(\Phi)=\frac{1}{b^{2}}(1-\cos (b \Phi)) .
$$

Here, $b$ is a free parameter which shall be related to the asymptotic curvature when the theory includes gravity. With this potential, new solutions connecting separate vacua are possible. One-soliton solutions for this potential read

$$
\Phi(y)=\frac{4}{b} \arctan e^{y}
$$




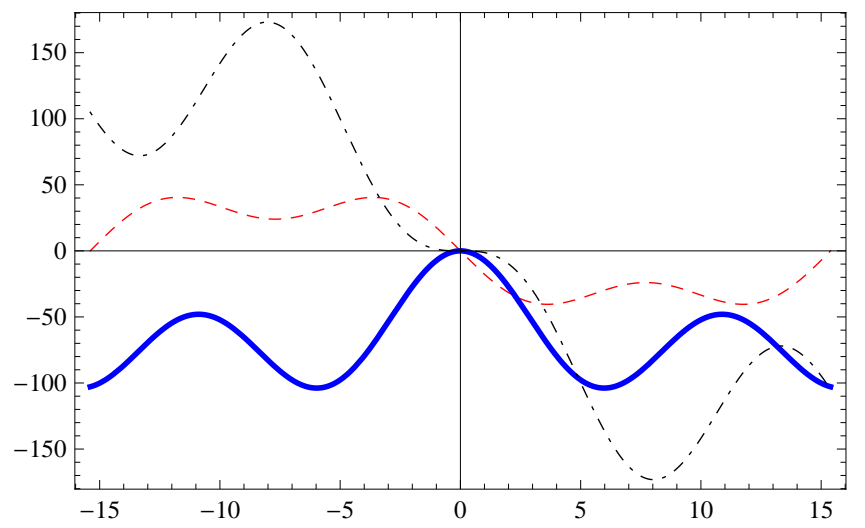

Figure 1: Modified background potential $\mathcal{V}(\Phi)$ (eq.14) for different values of $a=1 / 6 M^{3} b^{2}: a=1$ (dashed red line), $a=2$ (solid blue line), $a=3$ (dash-doted black line).

(we shall assume it static for simplicity). Now, in a gravitational background it is still possible that this bounce represents a legitimate brane-world. Considering the gravitational ansatz (2), the equations of motion (3) and (41) are compatible with this solution provided we somehow modify the potential functional. Taking into account eq.(5) we now obtain the superpotential

$$
\mathcal{W}(\Phi)=-\frac{4}{b^{2}} \cos \left(\frac{b}{2} \Phi\right)
$$

From (8) we get the following background potential

$$
\mathcal{V}(\Phi, \Pi)=-\exp \left(\Pi / \sqrt{12 M^{3}}\right) \cdot\left(\frac{4}{b^{2}} \sin ^{2}\left(\frac{b}{2} \Phi\right)+\frac{5}{2 M^{3} b^{4}} \cos ^{2} \frac{b}{2} \Phi\right)
$$

which can also be written as a function of $\Phi$ by means of

$$
\mathcal{V}(\Phi)=-\frac{4}{b^{2}}\left(\sin \frac{b}{2} \Phi\right)^{1 / 6 M^{3} b^{2}}\left(1+\left(\frac{5}{8 M^{3} b^{2}}-1\right) \cos ^{2} \frac{b}{2} \Phi\right),
$$

both exhibiting a highly nontrivial dependence on the scalar fields (see Fig. 1).

By writing the hamiltonian à la Bogomol'nyi, it can be deduced that $\mathcal{V}$ is consistent with the following relations among the warping, dilaton and superpotential

$$
\Pi=-\sqrt{3 M^{3}} \Lambda, \quad \Sigma=\Lambda / 4, \quad \Lambda^{\prime}=-\mathcal{W} / 12 M^{3}
$$

Solving the equations of motion, the explicit dependence of the dilaton field on the extra dimension is given by

$$
\Pi(y)=\frac{1}{\sqrt{3 M^{3}} b^{2}} \ln \cosh y
$$


from which we can readily obtain all the other field distributions of the background configuration.

As usual with dilaton configurations related to D-brane solutions, these functions are singular as $|y| \rightarrow \infty$. However, since the metric vanishes exponentially and both dilaton and warp factors operate by means of a negative exponential coupling, at the end of the day the model is free of divergences. Indeed, as we will see in the next Section, the effective action remains finite precisely thanks to the dilaton configuration. Note that the curvature of the metric with dilaton, as given by the Ricci scalar, now reads

$$
R(y)=-\left[8 \Lambda^{\prime \prime}(y)+18\left(\Lambda^{\prime}(y)\right)^{2}\right] \exp \left(\frac{\Pi(y)}{2 \sqrt{3 M^{3}}}\right),
$$

which for the background just obtained results in

$$
R(y)=16 a(\cosh y)^{a-2}\left(1-\frac{9}{2} \sinh ^{2} y\right),
$$

where $a=1 / 6 M^{3} b^{2}$. Since the dilaton contribution amounts to a redefinition of the effective four-dimensional Planck scale, eq.(18) exhibits a negative growing behavior far from the membrane. However, this problem disappears when we lift the metric solution up to $D=6$ [15], where the dilaton represents the radius of the new extra dimension as happens in type II string theory with $\mathrm{D} 4$ branes when lifted into $\mathrm{D}=11$ supergravity. As explained in [2], in six dimensions $d s_{6}^{2}=e^{3 A(y) / 2}\left(-d t^{2}+d x_{1}^{2}+d x_{2}^{2}+d x_{3}^{2}+d z^{2}\right)+d y^{2}$, where $z$ parametrize an extra $S^{1}$ direction, and this metric results everywhere regular for the solutions given above.

Studying as well the fluctuations of the metric about the above configuration, it is possible to see that this model supports a massless zero-mode of the gravitational field localized on the membrane even in the dilaton background. In order to prove the stability of the background solution, we would have to show that there are no negative mass solutions to the equations of motion of a perturbation $h_{\mu \nu}$ of the metric. Actually, a graviton massive spectrum appears starting from zero and presenting no gap. This can be easily seen by means of a supersymmetric type expression of the Schrodinger type operator which results after an appropriate change of variables and decomposition of the graviton field (see [2, 16] for details). The issue of the coupling of these massive modes to the brane has been analyzed in detail in [17]. 


\section{VECTOR AND TENSOR GAUGE FIELDS IN A WARPED SPACE WITH DILATON}

Now let us consider the system of five-dimensional electromagnetic $A_{N}$ and Kalb-Ramond $B_{N P}$ gauge fields coupled to the dilaton in a warped space-time. We will adopt the following $5 \mathrm{D}$ action

$$
S_{g}=\int d y d^{4} x \sqrt{-\operatorname{det} G_{A B}} e^{-\frac{\lambda}{2} \Pi}\left\{\frac{1}{12} e^{-\frac{\lambda}{2} \Pi} H_{M N P} H^{M N P}-\frac{1}{4} F_{M N} F^{M N}\right\}
$$

where $H_{M N P}=\partial_{[M} B_{N P]}$ and $F_{M N}=\partial_{[M} A_{N]}$.

Assuming that the gauge field energy density should not strongly modify the geometrical background, we can study the behavior of propagating modes in the background of the topological configuration studied in the last Section. In this respect, for example, Das et al. [18] derived an exact solution for the metric of a Randall-Sundrum (RS) approach with a Kalb-Ramond term (with no dilaton) and showed a negligible deviation from the pure RS solution without gauge fields. The new metric depends on the energy density of the KalbRamond field and goes smoothly to the RS solution in the limit of Kalb-Ramond energy density tending to zero. This scenario solves the hierarchy problem not just for the orbifold radius predicted by RS but for any value greater than the RS value. However, the important point here is that the Kalb-Ramond energy density is insignificant, amounting to $\sim 10^{-62}$. Indeed, this value matches remarkably well with the Kalb-Ramond energy density on the visible brane, calculated from the solution of the Kalb-Ramond field in a Randall Sundrum brane-world in a previous work [19]. In general, most of the attempts to stabilize 5D brane worlds by means of a scalar field in the bulk do not take into account the back-reaction of the scalar field on the background metric [2, 16, 17, 20] and those in order to compute the scalar back-reaction on the metric were unsuccessful except in a few special cases [21].

The dilaton $\Pi(y)$ couples exponentially with the kinetic terms of both gauge fields $B_{M N}$

and $A_{M}[22]$. This is related to the fact that the combination $\sqrt{G} \exp \Pi$ can be interpreted as a change in the integration measure. Still, different coupling constants $(\lambda$ and $\lambda / 2)$ are assigned to the 3-rank tensor $H_{M N P}$ and the electromagnetic field-strength $F_{M N}$ respectively [23]. This will be the origin of two different equations of motion for either gauge field as we now show.

It is easy to see that action (19) is invariant under gauge transformations $\delta B_{M N}=$ 
$\partial_{[M} \Omega_{N]}, \delta A_{M}=\partial_{M} \omega$. In five dimensions, the equations of motion for $B_{M N}$ and $A_{M}$ are given by

$$
\begin{aligned}
\frac{1}{\sqrt{-G}} \partial_{M}\left(G^{M R} G^{N P} F_{R P} \sqrt{-G} e^{-\frac{\lambda}{2} \Pi(y)}\right) & =0 \\
\frac{1}{\sqrt{-G}} \partial_{M}\left(G^{M R} G^{N S} G^{P Q} H_{R S Q} \sqrt{-G} e^{-\lambda \Pi(y)}\right) & =0
\end{aligned}
$$

where diag $G_{M N}=\left(e^{2 \Lambda} \eta_{\mu \nu}, e^{2 \Sigma}\right)$.

In order to solve these equations, we adopt the following gauge choices

$$
A^{5}=0, \partial_{\mu} A^{\mu}=0, B^{\mu 5}=0, \partial_{\mu} B^{\mu \nu}=0
$$

Next, we write down the equations in contravariant components $\left(T^{M N P \ldots}=\right.$ $\left.G^{M R} G^{N S} G^{P Q} \ldots T_{R S Q \ldots}\right)$ and separate the fifth from the other coordinates as follows

$$
A^{\mu}(x, y)=a^{\mu}(x) u(y), \quad B^{\mu \nu}(x, y)=b^{\mu \nu}(x) w(y) .
$$

Now, from eq.(20) we just get

$$
\begin{aligned}
& {\left[\square+\frac{1}{u f} \partial_{5}\left(f \partial^{5} u\right)\right] a^{\mu}=0} \\
& {\left[\square+\frac{1}{w g} \partial_{5}\left(g \partial^{5} w\right)\right] b^{\mu \nu}=0 .}
\end{aligned}
$$

Note that the metric deforms the otherwise trivial solutions of this system of equations through the warping functions and the dilaton field by means of the factors

$$
f(y)=\exp [4 \Lambda+\Sigma-\lambda \Pi / 2], \quad g(y)=f \exp [-\lambda \Pi / 2]
$$

acting on $u(y)$ and $w(y)$ respectively.

We may reduce the space of solutions to a rather special case. If we assume $u(y)=u_{0}$ and $w(y)=w_{0}$, normalizable zero-modes for the gauge fields can be obtained when $u_{0}$ and $w_{0}$ are (nonzero) constants. On the other hand, Kaluza-Klein modes result from the solution of the general case

$$
\partial_{5}\left(f \partial^{5} u\right)=-m_{A}^{2} f u, \quad \partial_{5}\left(g \partial^{5} w\right)=-m_{B}^{2} g w
$$

where $m_{A}^{2}, m_{B}^{2}$ are arbitrary constants representing the $4 \mathrm{D}$ squared bosons masses of vector and tensor gauge fields respectively. It means that $a^{\mu}=e^{i p x}$ with $\eta^{\mu \nu} p_{\nu} p_{\mu}=p^{2}=-m_{A}^{2}$, and $b^{\mu \nu}=e^{i k x}$ with $k^{2}=-m_{B}^{2}$. Below, we will show that these constants are indeed quantized 
eingenvalues of a Schrodinger-like equation. Furthermore, we will show that $u(y)=u_{0}$ and $w(y)=w_{0}$ are not just some special case but the unique zero-modes of the theory.

Explicitly, the most general $y$-dependent equations of motion from action (19) for the modified sine-Gordon potential (14) read

$$
\begin{gathered}
u^{\prime \prime}(y)+a\left(1-2 c_{1}\right) \tanh y u^{\prime}(y)+m_{A}^{2} \cosh ^{-a} y u(y)=0 \\
w^{\prime \prime}(y)+a\left(1-2 c_{2}\right) \tanh y w^{\prime}(y)+m_{B}^{2} \cosh ^{-a} y w(y)=0,
\end{gathered}
$$

where $c_{1}=\left(17+2 \lambda \sqrt{3 M^{3}}\right) / 4$ and $c_{2}=\left(17+4 \lambda \sqrt{3 M^{3}}\right) / 4$. We can see that the different dilaton coupling to the Maxwell and Kalb-Ramond fields is responsible for the different massive modes of vector and tensor bosons. Zero modes, however, are identical for both fields in this model. Nevertheless, as we will show later on, they are not necessarily localizable together.

\section{ANALYSIS OF THE GAUGE-BOSONS SPECTRA}

In this section we will discuss the existence of gauge field solutions to the model and their localization on the membrane. We can probe the localization of the gauge field modes by verifying that the corresponding action is finite. Note that from eq.(21) and eq.(22) it follows $H^{\mu \nu \rho}=h^{\mu \nu \rho} w(y)$ and $F^{\mu \nu}=f^{\mu \nu} u(y)$, so that

$$
\begin{aligned}
S_{g}[\mathrm{sol} .]= & \int d y u^{2}(y) e^{4 \Lambda(y)+\Sigma(y)-\lambda \Pi(y) / 2} \int d^{4} x \frac{1}{4} f_{\mu \nu} f^{\mu \nu}- \\
& \int d y \frac{1}{12} w^{2}(y) e^{4 \Lambda(y)+\Sigma(y)-\lambda \Pi(y)} \int d^{4} x h_{\mu \nu \alpha} h^{\mu \nu \alpha} .
\end{aligned}
$$

For constant $u(y)=u_{0}$ and $w(y)=w_{0}$, which satisfy the equations (26) and (27) for zero mass, this integral can be analytically proved to be finite. This shows that zero-modes associated with the Maxwell and Kalb-Ramond fields in the dilaton background can be localized on the four-dimensional space-time of a kink. Using the solutions found in eq.(11) and equations thereafter, we have

$$
\sim \int d y e^{4 \Lambda(y)+\frac{1}{4} \Lambda(y)+\frac{\lambda}{2} \sqrt{3 M^{3}} \Lambda(y)}
$$

which according to the solution

$$
\Lambda(y)=2 a \ln \text { sechy }
$$


(c.f. eq.(15) and eq.(16)) and the definition of constants $a$ and $c_{1}$, results in

$$
\int d y e^{c_{1} \Lambda}=\int d y \cosh ^{-2 a c_{1}} y<\infty
$$

provided $c_{1}>0$, namely $\lambda>-\frac{17}{2 \sqrt{3 M^{3}}}=\lambda_{0}$. If $c_{2}>0$, then $\lambda>\lambda_{0} / 2$ and there should be also localized zero-modes for the Kalb-Ramon field for any possible value of $\lambda$ in this interval.

Regarding Kaluza-Klein modes, eq.(26) and (27) could be numerically solved to have an idea about some particular cases. However, since an ordinary differential equation can always be put into its normal form, we can have a deeper insight on the whole problem. For this, let us then make the following transformation in eq.(26) (see e.g. [1, 2])

$$
u(y)=e^{-\alpha \Lambda / 2} U(z), \quad \frac{d z}{d y}=e^{-\beta \Lambda}
$$

where $\alpha$ and $\beta$ are arbitrary parameters. We now set $\alpha=c_{1}-1 / 4$ and $\beta=-1 / 4$ in order to eliminate the first derivative term in $U$ and to have a pure mass term. This brings eq.(26) into a simplest case of the Sturm-Liouville equation (with respect to a weight function 1). This is precisely a Schrodinger-like equation in the variable $z$

$$
\left[-\frac{d^{2}}{d z^{2}}+\mathfrak{V}(z)\right] U(z)=m_{A}^{2} U(z),
$$

where $\mathfrak{V}(z)=e^{-\Lambda / 2}\left(\frac{\alpha}{2} \Lambda^{\prime \prime}-\gamma \Lambda^{\prime 2}\right)$ and $\gamma=\frac{1}{4} \alpha\left(\frac{1}{2}-\alpha\right)$. If we now choose $a=2$ we arrive at the following expression for such analog non-relativistic potential

$$
\mathfrak{V}(z)=-2 \alpha \quad\left[1-(2 \alpha-1) \tan ^{2} z\right]
$$

which is a fully tractable function as we will see in what follows. Eq.(27) can be dealt with identically and we let it be considered in the next Sections.

\section{A. An exact subset of eigenstates}

Interestingly, the Schrodinger equation we have just identified has been recently discussed in the literature in a very different context. In [24] exact bound states have been analytically found for arbitrary $\alpha \geq 1 / 2$. Actually, bound states should be also possible for $\alpha \leq 0$ (besides localization of zero-modes precludes the region $\alpha \leq-1 / 4$ ) but this will be discussed in the next Section. Since $\mathfrak{V}(z)$ is defined within $-\pi / 2-2 \pi k<z<\pi / 2+2 \pi k, k \in$ 
$N$, bound eigenstates of eq.(33) have to vanish at the endpoints, i.e. $\left.U(z)\right|_{z= \pm(\pi / 2+2 \pi k)}=$ 0 . The reflection symmetry of the potential indicates that the eigenfunctions are exactly classified into two classes, symmetric and antisymmetric. These bound states are of course normalizable. However, in order to study normalization in the real bulk, we have to antitransform the solution back to the $u(y)$ space. It can be seen that there the only situation could arise in the case of positive $\alpha$ for large values of $y$; however, these correspond to values of $z$ close to $\pi / 2$ (and multiples) which are forbidden places for such a potential.

The symmetric eigenstates of eq.(33) can be generally written in an exact way in terms of Gauss hypergeometric functions

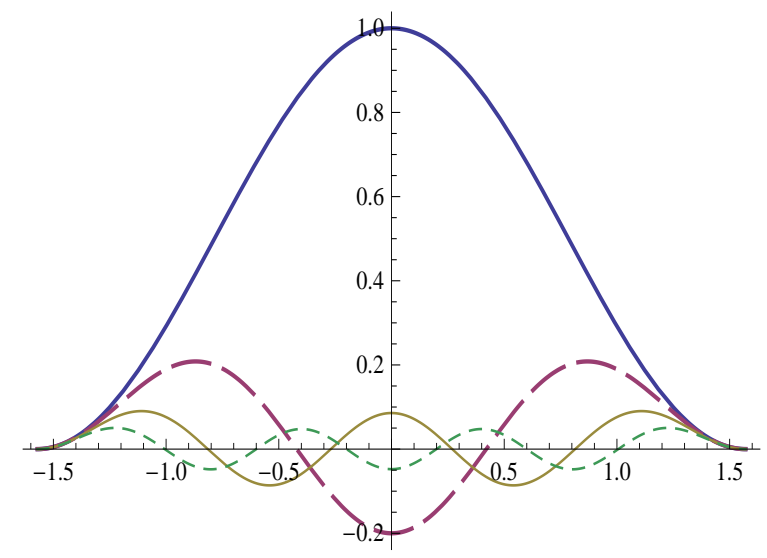

Figure 2: Symmetric $U_{2 n}(z)$ functions for $\alpha=1$ and $n=0$ (blue solid line), $n=1$ (red dashed line), $n=2$ (yellow solid line), $n=3$ (green short-dashed line).

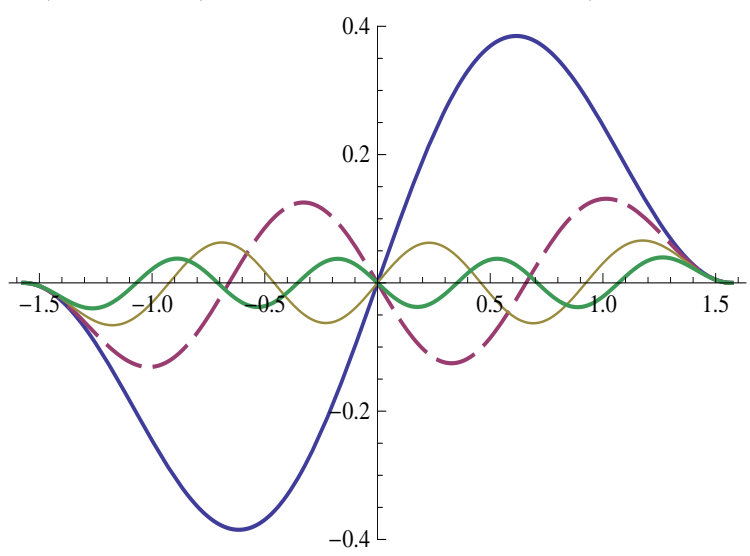

Figure 3: Antisymmetric $U_{2 n+1}(z)$ functions for $\alpha=1$ and $n=0$ (blue solid line), $n=1$ (red dashed line), $n=2$ (yellow thin-solid line), $n=3$ (green solid line).

$$
U_{2 n}(z ; \alpha)=A_{2 n}(\alpha)(\cos z)_{2}^{2 \alpha} F_{1}\left(-n, 2 \alpha+n, 2 \alpha+\frac{1}{2} ; \cos ^{2} z\right)
$$


where

$$
{ }_{2} F_{1}(a, b, c ; d)=1+\frac{a b d}{c 1 !}+\frac{a(a+1) b(b+1) d^{2}}{c(c+1) 2 !}+\ldots,
$$

and $A_{2 n}(\alpha)$ is a normalization constant.

In the case of anti-symmetric eigenstates, the exact solutions read

$$
U_{2 n+1}(z ; \alpha)=A_{2 n+1}(\alpha) \sin z(\cos z)_{2}^{2 \alpha} F_{1}\left(-n, 2 \alpha+n+1,2 \alpha+\frac{1}{2} ; \cos ^{2} z\right) \text {, }
$$

for $n=0,1,2,3, \ldots$ (see Fig. 2 and Fig. 33).

These functions are fully normalizable for $n \in N$ since the hypergeometric functions reduce to a polynomial of degree $n$ in $\cos ^{2} z$.

The corresponding analytical expression for both even and odd-indexed mass levels can be combined in a single expression

$$
m_{A}^{2}=l(4 \alpha+l), \quad l=0,1,2, \ldots
$$

for $l$ even and odd respectively. It is worth noting that this result exhibits an exact quantization condition on the massive modes of tensor and vector gauge fields inherited from the extra dimension.

In the original $y$ variable, these sets of solutions read

$$
u_{2 n}(y ; \alpha)=a_{2 n}(\alpha){ }_{2} F_{1}\left(-n, 2 \alpha+n, 2 \alpha+\frac{1}{2} ; \operatorname{sech}^{2} y\right)
$$

and

$$
u_{2 n+1}(y ; \alpha)=a_{2 n+1}(\alpha)(\tanh y){ }_{2} F_{1}\left(-n, 2 \alpha+n+1,2 \alpha+\frac{1}{2} ; \operatorname{sech}^{2} y\right),
$$

respectively, and are both finite polynomials in $\operatorname{sech}^{2} y ; a_{2 n}(\alpha)$ and $a_{2 n+1}(\alpha)$ are normalization constants.

Since eq.(33) involves an hermitian operator, for each value of $\alpha$ the spectrum is real and the above set of solutions is complete.

Now, regarding the second of eqs.(26), the procedure for the tensor massive modes can be performed on the same footing. By means of the transformation

$$
\frac{d z}{d y}=e^{-\beta_{2} \Lambda}, \quad w(y)=e^{-\alpha_{2} \Lambda / 2} W(z)
$$

we obtain similar, though not identical, modes for the Kalb-Ramond field. The difference results from the change $\alpha \rightarrow \alpha_{2}=4+\lambda \sqrt{3 M^{3}}$ which modifies the spectrum and the 


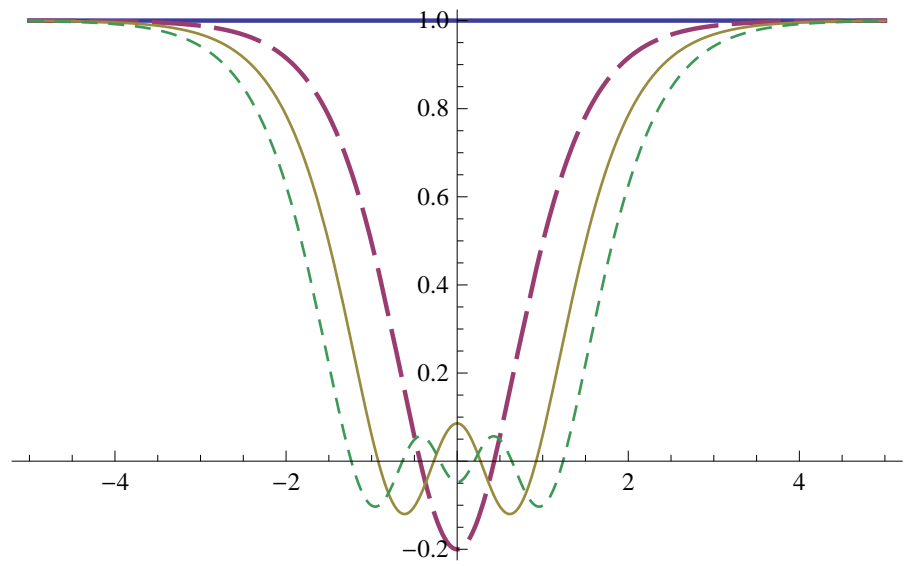

Figure 4: Symmetric solutions $u_{2 n}(y)$ for $\alpha=1$ and $n=0$ (blue solid line), $n=1$ (red dashed line), $n=2$ (yellow thin solid line), $n=3$ (green short-dashed line); $n=0$ corresponds to the massless mode.

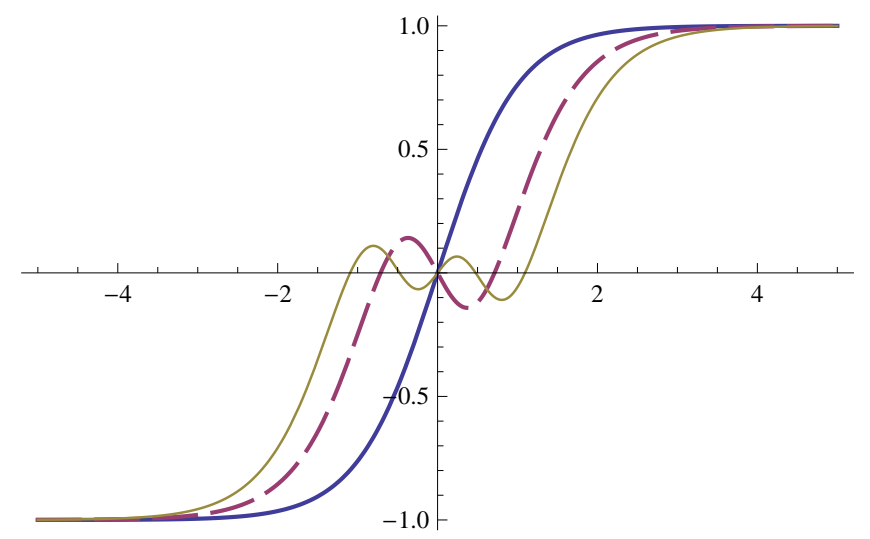

Figure 5: Antisymmetric solutions $u_{2 n+1}(y)$ for $\alpha=1$ and $n=0$ (blue solid line), $n=1$ (red dashed line), $n=2$ (yellow thin solid line).

eigenstates. This can be directly seen from the set of equations above by replacing $\alpha$ by $\alpha_{2}$. We include them for the sake of completeness

$$
w_{2 n}\left(y ; \alpha_{2}\right)=b_{2 n}\left(\alpha_{2}\right){ }_{2} F_{1}\left(-n, 2 \alpha_{2}+n, 2 \alpha_{2}+\frac{1}{2} ; \operatorname{sech}^{2} y\right)
$$

and

$$
w_{2 n+1}\left(y ; \alpha_{2}\right)=b_{2 n+1}\left(\alpha_{2}\right)(\tanh y){ }_{2} F_{1}\left(-n, 2 \alpha_{2}+n+1,2 \alpha_{2}+\frac{1}{2} ; \operatorname{sech}^{2} y\right),
$$

where $a_{n}\left(\alpha_{2}\right)$ and $b_{n}\left(\alpha_{2}\right)$ are the normalization constants of the KR modes, and the corresponding spectrum is

$$
m_{B}^{2}=l\left(4 \alpha_{2}+l\right), \quad l=0,1,2, \ldots .
$$

for $l$ even and odd respectively. 


\section{THE COMPLETE ANALYSIS}

There is an important question to be clarified regarding the solution of our problem. Often in the literature, the dimensional reduction procedure leads to the above situation in which the original differential equation for the quantum fields is mapped onto a Schrodinger equation by means of a transformation like (32). The calculation is then performed but not rarely without due care of the misleading non-relativistic quantum-mechanical aspect of the problem at hand. Indeed, following [24], in the last section we have limited our analysis to solutions that are constrained by the quantum-mechanical features of eq.(33) and (34). However, although the differential equation for $U$ is given by a Schrodinger operator, in the present context $\left[-\frac{d^{2}}{d z^{2}}+\mathfrak{V}(z)\right]$ is not of true hamiltonian nature, as it is the case in actual Quantum Mechanics. This implies that a quantum-mechanical reasoning may be physically incomplete, or even wrong in several aspects. In fact, in the real transverse physical space the equation of motion is (26) which is not Schrodinger at all. Furthermore, for any $\alpha<0$ it can be verified that the $m^{2}=0$ eigenvalue would not even be allowed by the potential because the corresponding 'energy' $(E=2 \alpha)$ is below its minimum. It would be in principle a wrong restriction since the zero-modes of eq.(26) do exist and are simply given by $u=$ cons, which is consistent with $U=A \cos ^{2 \alpha} z$ for any value of $\alpha \in R$ provided the hypergeometric functions are well defined (analogously for $w=$ cons and $W=B \cos ^{2 \alpha_{2}} z$ ). Thus, the hamiltonian criterion is not justified since eq. (333) does not describe any nonrelativistic quantum particle. Therefore, the existence and localization of a Maxwell zeromode is possible in a wider range than just $\alpha \geq 1 / 2$ as implicitly assumed in the solutions found in the previous Section (see e.g. Figs. 6,7] and Figs. 8,9). In order to constrain the values of the coupling constant we shall take into account the analyticity of the solutions in the $y$ space, and other physical reasons such as localization of zero-modes and the absence of tachyons in the theory. The Kalb-Ramond field will be analyzed as well by means of $\alpha_{2}$.

Let us emphasize that the spectrum given by eq.(38) and (39) is complete provided one imposes boundary conditions consistent with the hamiltonian problem, namely, vanishing solutions $U(z)$ at $z= \pm \pi / 2$ (in the $z$-space). Since it is not enough to fully describe what is going on in the $y$-space, we need to release these boundary conditions. Actually, in order to study all the relevant solutions of the Sturm-Liouville problem (33) related to the original differential equation, we should admit all the boundary conditions compatible with finiteness 


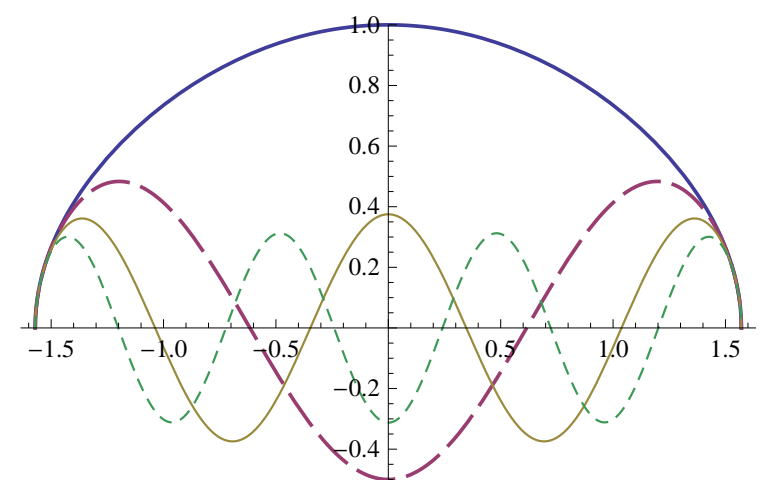

Figure 6: Symmetric $U(z)$ type functions for $\alpha=1 / 4$ and $n=0$ (blue solid line), $n=1$ (red dashed line), $n=2$ (yellow thin-solid line), $n=3$ (green short-dashed line); $n=0$ is for the massless mode.

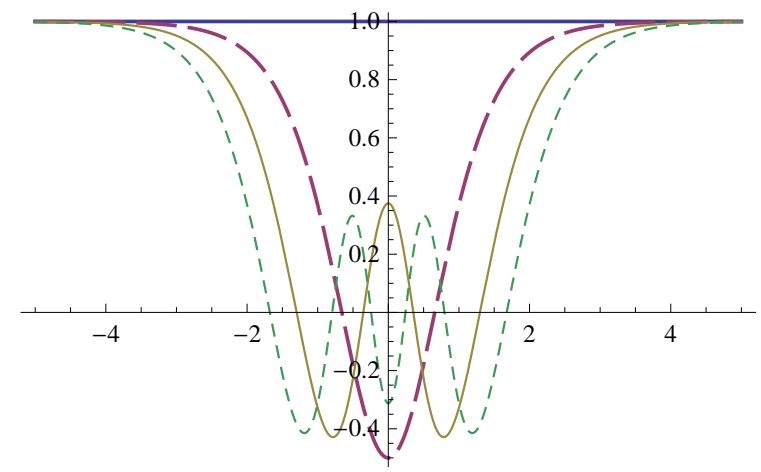

Figure 7: Symmetric $u_{\text {even }}^{(1)}(y)$ solutions for $\alpha=1 / 4$ and $n=0$ (blue solid line), $n=1$ (red dashed line), $n=2$ (yellow thin-solid line), $n=3$ (green short-dashed line); the constant corresponds to the massless gauge mode $5 \mathrm{D}$ factor $(n=0$, solid blue).

of the $u, w$ functions in the $y$ space. For this, we should first relax nullification at $z= \pm \pi / 2$ and just require convergence in the open interval $(-\pi / 2, \pi / 2)$ admitting divergencies at the end points provided we get finite values after mapping back to the $y$ space. This can be done by completely relaxing the parameters in the Schrodinger equation above irrespective of quantum interpretations in the $z$-space. We will thus just focus on $u(y)$ solutions which are the actual and direct 5D factors of physical gauge fields.

\section{A. Full gauge-field spectrum}

To start, note that in SectionIVA the so-called second solution of the differential equation has been just disregarded because it does not vanish at $z= \pm \pi / 2$ as expected for quantum 


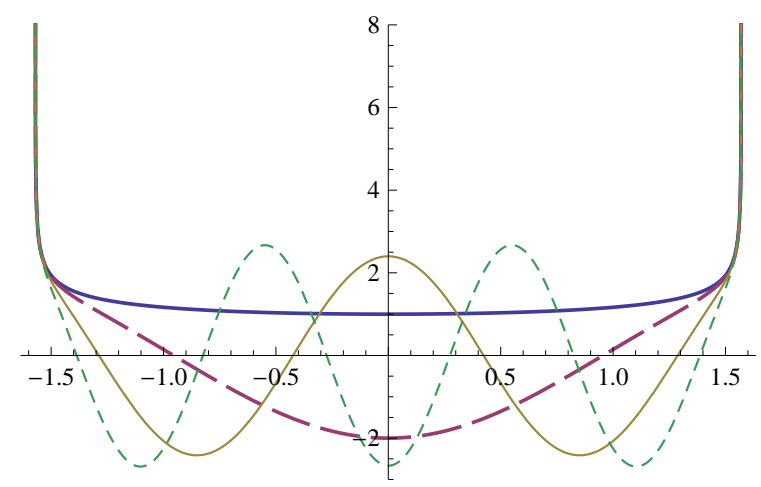

Figure 8: Symmetric $U(z)$ functions for $\alpha=-1 / 8$ and $n=0$ (blue solid), $n=1$ (red dashed), $n=2$ (yellow thin-solid), $n=3$ (green short-dashed). Note that all of them are divergent at $z= \pm \pi / 2$.

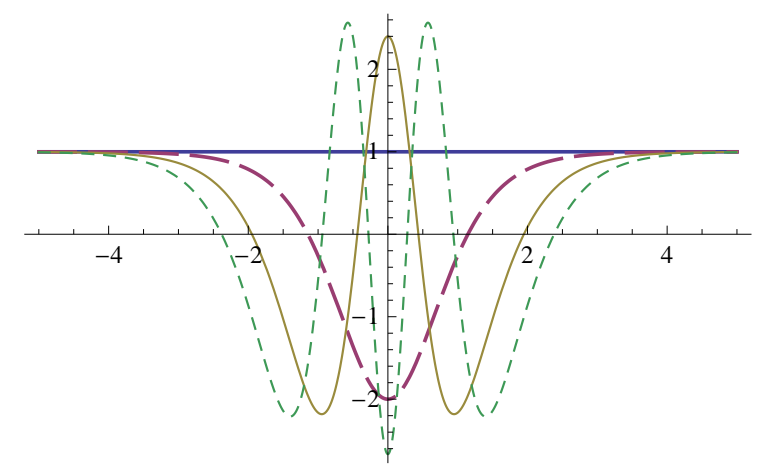

Figure 9: Symmetric $u_{\mathrm{even}}^{(1)}(y)$ solutions for $\alpha=-1 / 8$ and $n=0$ (blue solid), $n=1$ (red dashed), $n=2$ (yellow thin-solid), $n=3$ (green short-dashed). All are fully convergent in the $y$ space despite the divergences in the $z$ coordinate. The constant corresponds to $n=0$ (solid-blue).

mechanical bound-states. Now, as a result of our discussion above, we will hereafter include this kind of solutions

\section{Symmetric eigenstates}

As a matter of fact, in the case of even states the complete set of eigenfunctions to be considered is not just given by eq.(38) but by both

$$
u_{\text {even }}^{(1)}(y ; \alpha, n)=a_{\text {even }}^{(1)}(\alpha, n){ }_{2} F_{1}\left(-n, 2 \alpha+n, 2 \alpha+\frac{1}{2} ; \operatorname{sech}^{2} y\right),
$$

and

$$
u_{\text {even }}^{(2)}(y ; \alpha, n)=a_{\text {even }}^{(2)}(\alpha, n)(\operatorname{sech} y)^{1-4 \alpha}{ }_{2} F_{1}\left(\frac{1}{2}+n,-2 \alpha+\frac{1}{2}-n,-2 \alpha+\frac{3}{2} ; \operatorname{sech}^{2} y\right),
$$




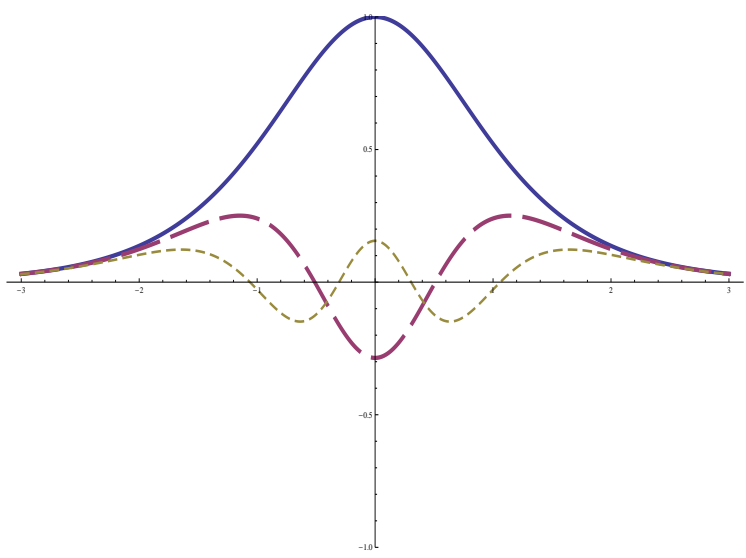

Figure 10: Second-symmetric solutions $u_{\text {even }}^{(2)}(y)$ for $\alpha=-1 / 8$ and $n=-1 / 2-p$ where $p=0$ (blue solid), $p=1$ (red dashed), $p=2$ (yellow short-dashed).

in order to cover the whole space of solutions of the differential operator. Now, notice that nothing prevents $n$ from being a full real number independently of $\alpha$, which is also in $R$. The only restriction concerns the points where the Gauss functions are not well defined, namely $\alpha=-\frac{1}{4},-\frac{3}{4},-\frac{5}{4}, \ldots$ and $\alpha=\frac{3}{4}, \frac{5}{4}, \frac{7}{4}, \ldots$, for the first and second functions respectively. In fact, in the second case $\alpha>1 / 4$ is already excluded due to the $(\operatorname{sech} y)^{1-4 \alpha}$ factor and, in both, $\alpha \leq-1 / 4$ is unimportant for we are not interested in values which do not admit a localizable zero-mode.

Still, we can only accept continuous and differentiable gauge fields, so we have to analyze their behavior at the origin (Gauss hypergeometric functions are convergent at any point else). In case of eq.(44), right and left derivatives at $y=0$ are

$$
\left.\frac{d u_{\text {even }}^{(1)}}{d y}\right|_{y=0^{ \pm}}= \pm \frac{\sqrt{\pi} 4 n(n+2 \alpha) \Gamma(3 / 2+2 \alpha)}{(1+4 \alpha) \Gamma(1-n) \Gamma(1+n+2 \alpha)} .
$$

Thus, derivatives are continuous only if

$$
n=0,1,2, \ldots, \quad \text { or } \quad n+2 \alpha=0,-1,-2, \ldots
$$

It shows that we can obtain well-behaved $u_{\text {even }}^{(1)}$ solutions (and localizable zero modes) not only for $n \in N$ but also for $n \in R$ provided it is related to $\alpha$ by $n=-k-2 \alpha, k \in N$, with $\alpha \in R-\{\alpha \leq-1 / 4\}$. In fact, solutions $u_{\text {even }}^{(1)}(y)$ are symmetric under the change $n+2 \alpha \leftrightarrows-n$. Thus, in any such cases solutions will be identical.

In case of eq.(45), right and left derivatives at $y=0$ are

$$
\left.\frac{d u_{\mathrm{even}}^{(2)}}{d y}\right|_{y=0^{ \pm}}= \pm \frac{\sqrt{\pi}\left(4 n^{2}+8 n \alpha+4 \alpha-1\right) \Gamma(5 / 2-2 \alpha)}{(4 \alpha-3) \Gamma(3 / 2+n) \Gamma(3 / 2-n-2 \alpha)} .
$$




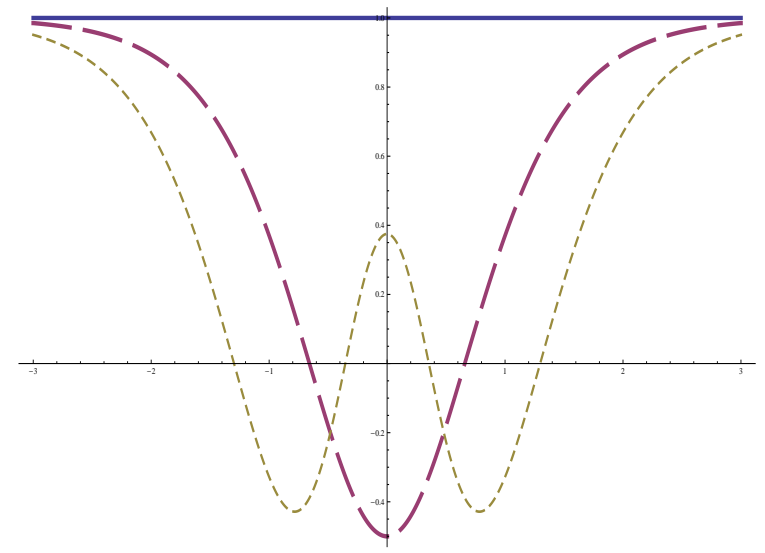

Figure 11: Second-symmetric solutions $u_{\mathrm{even}}^{(2)}(y)$ for $\alpha=1 / 4$ and $n=-1 / 2-p$ where $p=0$ (blue solid), $p=1$ (red dashed), $p=2$ (yellow short-dashed). Note the presence of a zero-mode for $p=0$ (blue solid line).

These derivatives are continuous provided

$$
n=-1 / 2,-3 / 2, \ldots, \quad \text { or } \quad n+2 \alpha=1 / 2,3 / 2, \ldots
$$

and we can also have well-behaved $u_{\text {even }}^{(2)}$ solutions (and localizable zero modes) for $n$ real when $n=-k / 2$ or $n=k / 2-2 \alpha, k \in N_{0}$, with $\alpha \in(-1 / 4<\alpha \leq 1 / 4]$. See Figs. [10]11. Solutions $u_{\text {even }}^{(2)}(y)$ are also symmetric under the change $n+2 \alpha \leftrightarrows-n$.

In order to identify symmetric zero-modes, we analyze the even spectrum, which is given by

$$
m^{2}=4 n(n+2 \alpha)
$$

From this we can pick the following possibilities: $u_{\mathrm{even}}^{(1)}(n=0, \alpha>-1 / 4)$, and $u_{\mathrm{even}}^{(1)}(n=$ $-2 \alpha, \alpha>-1 / 4)$, and $u_{\text {even }}^{(2)}(n=0, \alpha=1 / 4)$ and $u_{\text {even }}^{(2)}(n=-1 / 2, \alpha=1 / 4)$ where analyticity together with the localization restriction have been assumed. Note that these are in fact constants for every value of $\alpha$, as already pointed out.

If we now look for a massive spectrum free of tachyons, other constraints will restrict the space of legal solutions. Since the even spectrum is given by eq.(150), tachyons are avoided only in the following cases

$$
\begin{aligned}
& (n=1, \alpha>-1 / 2),(n=2, \alpha>-1), \ldots \\
& (n=-1-2 \alpha, \alpha>-1 / 2),(n=-2-2 \alpha, \alpha>-1), \ldots
\end{aligned}
$$




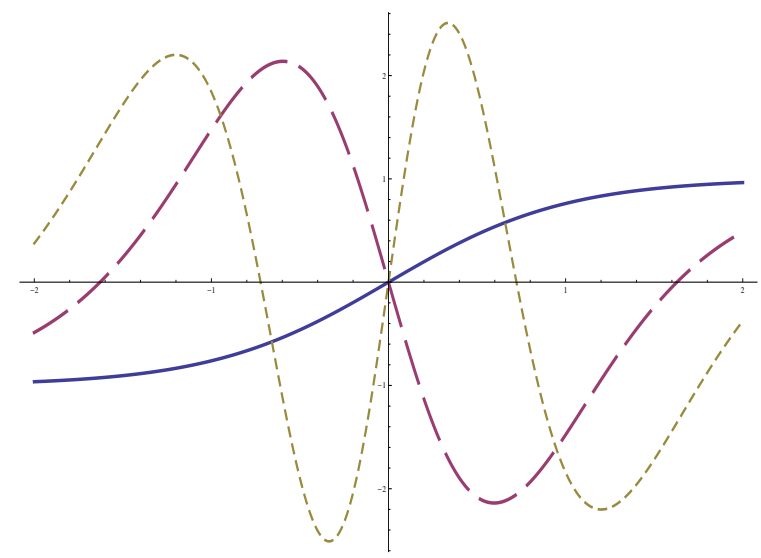

Figure 12: Antisymmetric solution $u_{\mathrm{AS}}^{(1)}(y)$ for $\alpha=-1 / 8$ and $n=0$ (blue solid), $n=1$ (red dashed), $n=2$ (yellow short-dashed line).

for $u_{\mathrm{even}}^{(1)}$, and

$$
\begin{aligned}
& (n=-1 / 2, \alpha<1 / 4),(n=-3 / 2, \alpha<3 / 4),(n=-5 / 2, \alpha \leq 5 / 4), \ldots \\
& (n=1 / 2-2 \alpha, \alpha<1 / 4),(n=3 / 2-2 \alpha, \alpha<3 / 4),(n=5 / 2-2 \alpha, \alpha<5 / 4), \ldots
\end{aligned}
$$

for $u_{\mathrm{even}}^{(2)}$. In each case, these additional constraints have to be considered on top of the corresponding analyticity restrictions shown above. This demonstrates that there is no risk of tachyons among analytic symmetric eigenstates when localized zero modes are demanded in the theory. There is however an important remark to be done in this respect when just one of the gauge fields is demanded to deposit zero modes on the brane. We will come to this point in Section $\mathrm{VB}$.

\section{Antisymmetric eigenstates}

Regarding antisymmetric eigenfunctions, the complete set of solutions is

$$
u_{\mathrm{AS}}^{(1)}(y ; \alpha, n)=a_{\mathrm{AS}}^{(1)}(\alpha, n)(\tanh y){ }_{2} F_{1}\left(-n, 2 \alpha+n+1,2 \alpha+\frac{1}{2} ; \operatorname{sech}^{2} y\right),
$$

and

$$
\begin{aligned}
u_{\mathrm{AS}}^{(2)}(y ; \alpha, n)= & a_{\mathrm{AS}}^{(2)}(\alpha, n)(\operatorname{sech} y)^{1-4 \alpha}(\tanh y) \\
& { }_{2} F_{1}\left(1 / 2+n,-2 \alpha-n+\frac{1}{2},-2 \alpha+\frac{3}{2} ; \operatorname{sech}^{2} y\right) .
\end{aligned}
$$




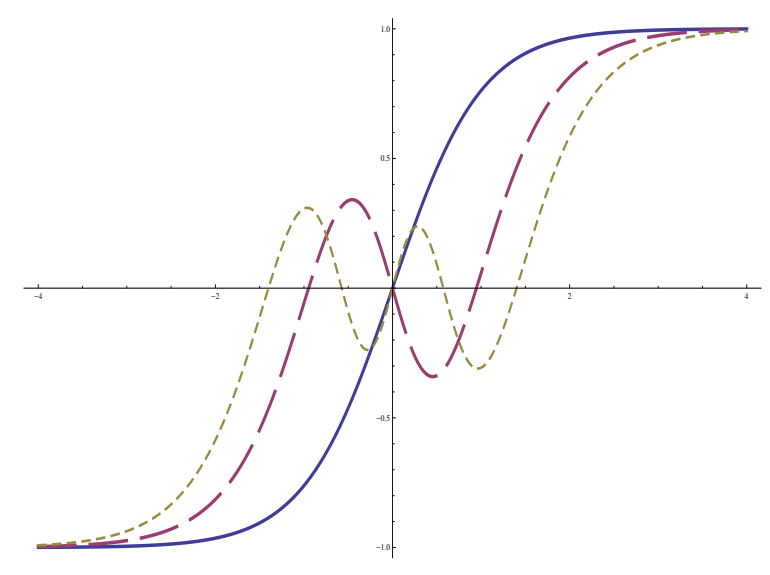

Figure 13: Antisymmetric solution $u_{\mathrm{AS}}^{(1)}(y)$ for $\alpha=3 / 8$ and $n=0$ (blue solid), $n=1$ (red dashed), $n=2$ (yellow short dashed line).

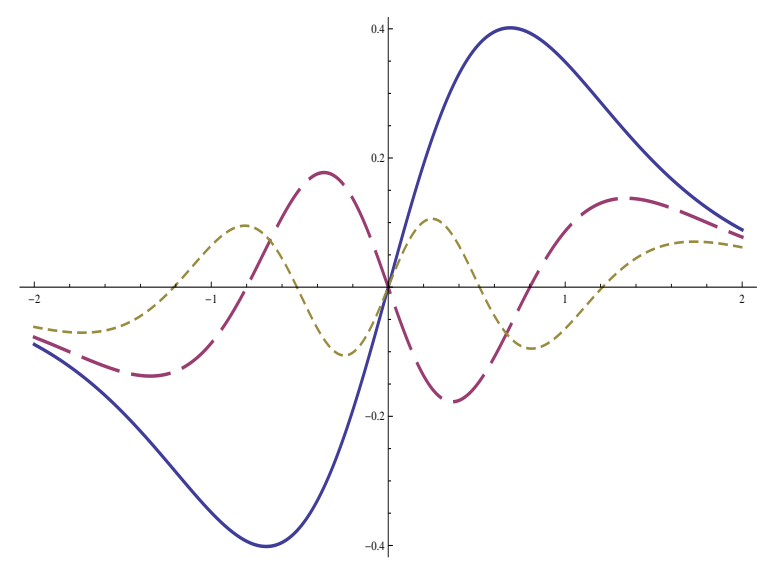

Figure 14: Second-antisymmetric solution $u_{\mathrm{AS}}^{(2)}(y)$ for $\alpha=-1 / 5$ and $n=-3 / 2-p$ where $p=0$ (blue solid), $p=1$ (red dashed), $p=2$ (yellow short-dashed line).

The first of these is not defined for $\alpha=-\frac{1}{4},-\frac{3}{4},-\frac{5}{4}, \ldots$ and the second for $\alpha=\frac{3}{4}, \frac{5}{4}, \frac{7}{4}, \ldots$. As before, in the second case $\alpha>1 / 4$ is excluded due to the divergency of $(\operatorname{sech} y)^{1-4 \alpha} \tanh y$, while $\alpha \leq-1 / 4$ is unimportant in both cases for we will not be interested in values which do not admit localizable zero-modes.

Unlike even functions, antisymmetric functions can be discontinuous at the origin. Actually, we have

$$
u_{\mathrm{AS}}^{(1)}\left(y=0^{ \pm}\right)= \pm \frac{\sqrt{\pi} \Gamma(1 / 2+2 \alpha)}{\Gamma(-n) \Gamma(1+n+2 \alpha)}
$$

although left and right derivatives coincide

$$
\left.\frac{d u_{\mathrm{AS}}^{(1)}}{d y}\right|_{y=0^{ \pm}}={ }_{2} F_{1}\left(-n, 2 \alpha+n+1,2 \alpha+\frac{1}{2} ; 1\right) .
$$




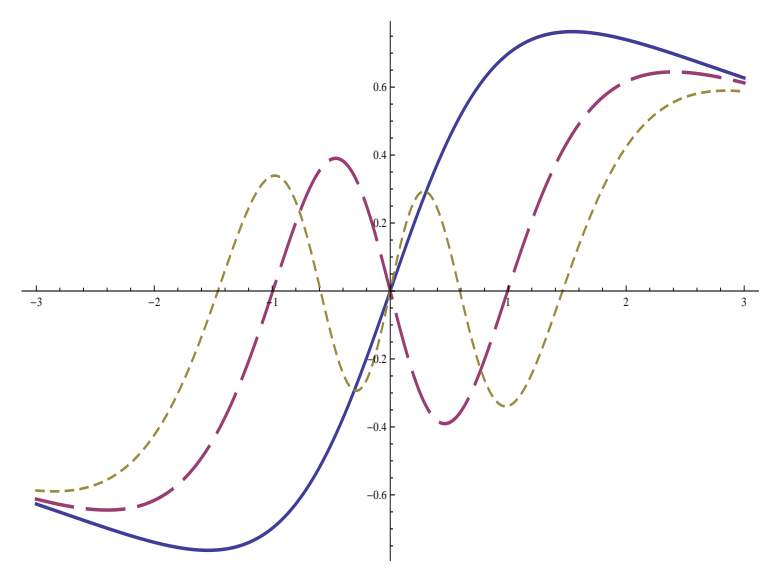

Figure 15: Second-antisymmetric solution $u_{\mathrm{AS}}^{(2)}(y)$ for $\alpha=1 / 5$ and $n=-3 / 2-p$ where $p=0$ (blue solid), $p=1$ (red dashed), $p=2$ (yellow short-dashed line).

Thus, in order to avoid discontinuities of the gauge field we require

$$
n=0,1,2, \ldots, \quad \text { or } \quad n+2 \alpha=-1,-2, \ldots .
$$

and $\alpha \neq-\frac{1}{4}$ which is already forbidden.

The second antisymmetric solution can also be discontinuous depending on the value of the parameters. The analytical right and left expressions are

$$
u_{\mathrm{AS}}^{(2)}\left(y=0^{ \pm}\right)= \pm \frac{\sqrt{\pi} \Gamma(3 / 2-2 \alpha)}{\Gamma(3 / 2+n) \Gamma(1 / 2-n-2 \alpha)},
$$

so that these eigenfunctions are continuous only for

$$
n=-3 / 2,-5 / 2, \ldots, \quad \text { or } \quad n+2 \alpha=1 / 2,3 / 2, \ldots
$$

and $\alpha \neq 3 / 4,5 / 4, \ldots$, all of which are above the region of convergence of the hyperbolic overall factor. The exact derivatives of this family of eigenstates are

$$
\left.\frac{d u_{\mathrm{AS}}^{(2)}}{d y}\right|_{y=0^{ \pm}}={ }_{2} F_{1}\left(3 / 2+n,-2 \alpha-n+\frac{1}{2},-2 \alpha+\frac{3}{2} ; 1\right)
$$

which are well defined for all $\alpha \neq \frac{3}{4}, \frac{5}{4}, \ldots$ (these are all bigger than $1 / 4$ and thus outside the range of convergence of $u_{\mathrm{AS}}^{(2)}(y ; \alpha, n)$ as already signaled).

In the antisymmetric case, the mass spectrum is given by

$$
m^{2}=(2 n+1)(2 n+1+4 \alpha)
$$

so zero-modes are not possible considering the analyticity restrictions of these eigenfunctions. 
In order to avoid tachyons, besides conditions for existence and localization, in the case of $u_{\mathrm{AS}}^{(1)}$ we shall require

$$
\begin{aligned}
& (n=0, \alpha>-1 / 4),(n=1, \alpha>-3 / 4),(n=2, \alpha>-5 / 4), \ldots \\
& (n=-1-2 \alpha, \alpha>-1 / 4),(n=-2-2 \alpha, \alpha>-3 / 4), \ldots
\end{aligned}
$$

while, regarding $u_{\mathrm{AS}}^{(2)}$, the non-tachyon constraints are

$$
\begin{aligned}
& (n=-3 / 2, \alpha<1 / 2),(n=-5 / 2, \alpha<1), \ldots \\
& (n=1 / 2-2 \alpha, \alpha),(n=3 / 2-2 \alpha, \alpha), \ldots .
\end{aligned}
$$

Tachyons are again impossible considering the analyticity constraints of the functions and the restrictions on $\alpha$ due to localization of zero modes.

All this analysis can be straightforwardly repeated for the Kalb-Ramond field just by changing the parameter $\alpha \rightarrow \alpha_{2}$.

\section{B. Phase interpretation picture}

The present field theory can be classified into different phase configurations depending on the value of the dilaton coupling constant. Since the exact value of this coupling constant should be specified by an ascendant theory we will again consider the whole range for this analysis. In order to characterize the possible different phases in the theory we can use the shape of the analog potential functions (34) appearing in eq.(33).

In the parameter region $\alpha \geq 1 / 2$ or $\alpha \leq 0$, eq.(34) defines a bounding analog potential $\mathfrak{V}(z) \geq 0$ (see Fig. 16). On the contrary, in the complementary region of the $\alpha$ parameter, namely $0<\alpha<1 / 2, \mathfrak{V}(z)$ is negative allowing for a different physical picture (see Fig. 17). Even in this case, when the mass is zero we get as announced a constant value for the $u$ solution in the $y$ space (note that this legal mode is forbidden in a strictly hamiltonian interpretation of the problem).

If $\alpha \leq-1 / 4$, then $\lambda \leq \lambda_{0}$ and a localized gauge zero-mode is impossible as pointed out earlier. However, if $\alpha_{2} \leq-1 / 4$ the coupling is $\lambda \leq \lambda_{0} / 2$ and then, although no Kalb-Ramond zero-mode is localized, there can be a Maxwell zero-mode deposited on the membrane. If

$0<\alpha<1 / 2$, then $\frac{16}{17} \lambda_{0}<\lambda<\frac{14}{17} \lambda_{0}$ which lies also between $\lambda_{0}<\lambda<\lambda_{0} / 2$ and again no Kalb-Ramond but just a Maxwell zero-mode has a (finite) contribution to the effective 


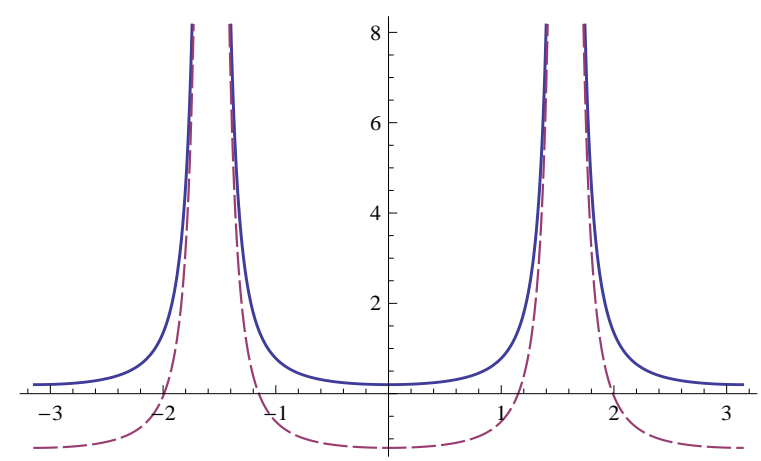

Figure 16: $\mathfrak{V}_{A}(z)$ for $\alpha=-1 / 10$ (solid blue) and $\alpha=1 / 2+1 / 10$ (red dashed).

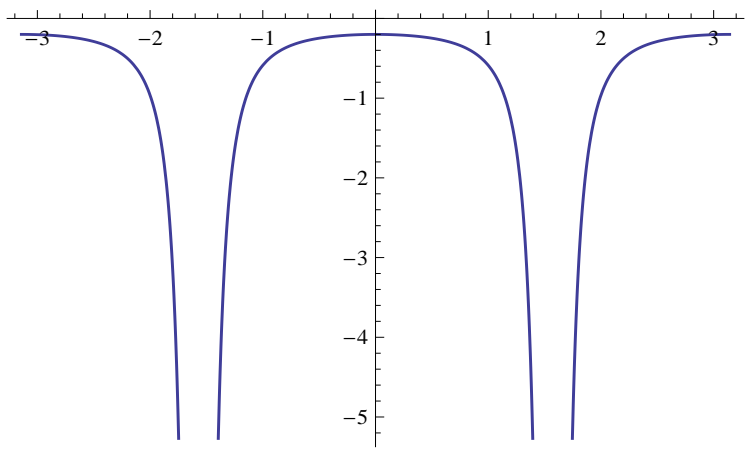

Figure 17: $\mathfrak{V}_{A}(z)$ for $\alpha=1 / 2-1 / 10$.

action. If $0<\alpha_{2}<1 / 2$, then both zero-modes should be localizable. Note that, if the energy criterion was valid, a Maxwell zero-mode would only exist for $\lambda>\frac{16}{17} \lambda_{0}$ and, on the same footing a localized zero-mode for the Kalb-Ramond field would only exist provided $\alpha_{2}>0$, namely $\lambda>\frac{8}{17} \lambda_{0}$.

In terms of the dilaton coupling constant, the simultaneous analysis of both gauge fields results in the following conclusion: a gauge zero mode exists and is localizable provided $\lambda>\lambda_{0}$ with the undermentioned details. $i$ ) In $\frac{16}{17} \lambda_{0}<\lambda<\frac{14}{17} \lambda_{0}$ the theory possess a localized Maxwell zero-mode and a tower of normalizable massive Maxwell modes resulting from an analog potential $\mathfrak{V}_{A}(z)>0$, together with normalizable Kalb-Ramond massive states related to an analog potential $\mathfrak{V}_{B}(z)<0$. ii) In $\frac{14}{17} \lambda_{0}<\lambda<\frac{1}{2} \lambda_{0}$ there is a Maxwell zero-mode and normalizable massive Maxwell modes related to $\mathfrak{V}_{A}(z)<0$ together with normalizable Kalb-Ramond massive states related to $\mathfrak{V}_{B}(z)<0$. iii) In $\frac{1}{2} \lambda_{0}<\lambda<\frac{8}{17} \lambda_{0}$ a Kalb-Ramond zero-mode is also deposited on the previous configuration. $i v$ ) In $\frac{8}{17} \lambda_{0}<\lambda<\frac{7}{17} \lambda_{0}$ the model presents a Maxwell zero-mode and massive Maxwell modes as resulting from $\mathfrak{V}_{A}(z)>0$, 


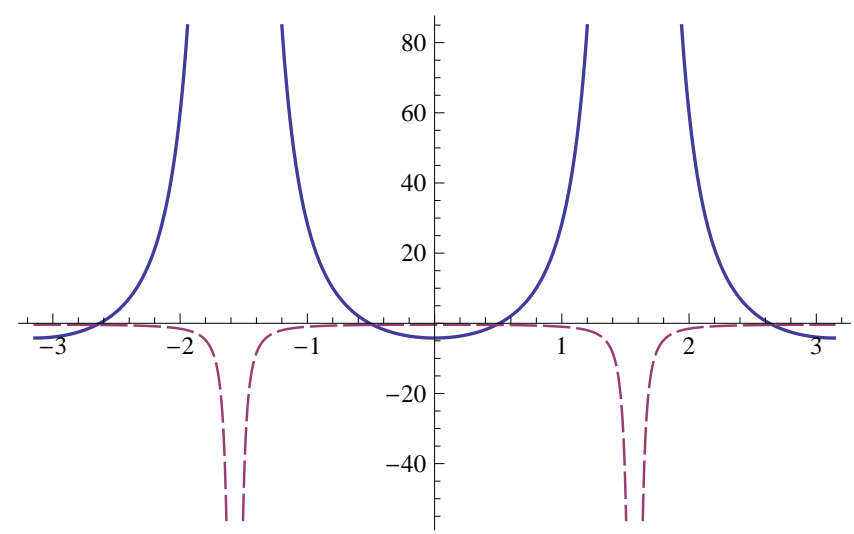

Figure 18: $\mathfrak{V}_{A}(z)$ (solid) and $\mathfrak{V}_{B}(z)$ (dashed) for $\alpha_{2}=1 / 5$. It corresponds to a dilaton coupling constant in $\frac{8}{17} \lambda_{0}<\lambda<\frac{7}{17} \lambda_{0}$.

together with a Kalb-Ramond zero-mode and massive Kalb-Ramond states coming from a potential $\mathfrak{V}_{B}(z)<0$. v) If $\lambda>\frac{7}{17} \lambda_{0}$, both Maxwell and Kalb-Ramond zero-modes are localized and normalizable Kaluza-Klein eigenstates can be related to $\mathfrak{V}_{A, B}(z)>0$.

We thus conclude that depending on the value of the dilaton coupling the theory sits in one among several phases, which are given by the global sign of the analog potentials defined in transverse space. The two potentials change dramatically as $\alpha$ (or $\alpha_{2}$ ) take values on one side or another of 0 and $1 / 2$. Thus, from $\lambda=\frac{16}{17} \lambda_{0}-\epsilon$ to $\lambda=\frac{16}{17} \lambda_{0}+\epsilon$, the analog potential flips from Fig. 16 (solid) to Fig. 17, and for $\lambda=\frac{14}{17} \lambda_{0}+\epsilon$ instead of $\frac{14}{17} \lambda_{0}-\epsilon$ the analog potential is that of Fig. 16 (dashed) instead of Fig. 17. Analogously, from $\frac{8}{17} \lambda_{0}+\epsilon$ to $\frac{8}{17} \lambda_{0}-\epsilon$ the potential for $\mathrm{KR}$ modes $\mathfrak{V}_{B}(z)$ would invert its concavity, but it would be back the same for $\lambda$ above $\frac{7}{17} \lambda_{0}$.

Particularly interesting is the interval $\frac{8}{17} \lambda_{0}<\lambda<\frac{7}{17} \lambda_{0}$ where Maxwell and Kalb-Ramond fields experience totally different analog potentials at the same time (see Fig. 18) and both have zero-modes localized on the membrane.

Finally, it is worth noting that if one just demands the theory to possess a localized Maxwell zero-mode but no such condition is put on the Kalb-Ramond field, there is a full segment of possible values of the dilaton coupling constant where several kinds of gauge messengers could co-exist. Specifically, in the interval going from $\lambda_{0}$ to $\lambda_{0} / 2$, namely $\alpha \in$ $(-1 / 4,15 / 8]$. When $\lambda>\lambda_{0}$, we can write $\alpha=-1 / 4+\epsilon$ and $\alpha_{2}=-9 / 2+2 \epsilon$. In this cases Kaluza-Klein eigenstates are well defined for both fields but some Kalb-Ramond modes are compatible with $m_{B}^{2}<0$. For example, when $\epsilon$ is sufficiently small, the eigenfunctions 


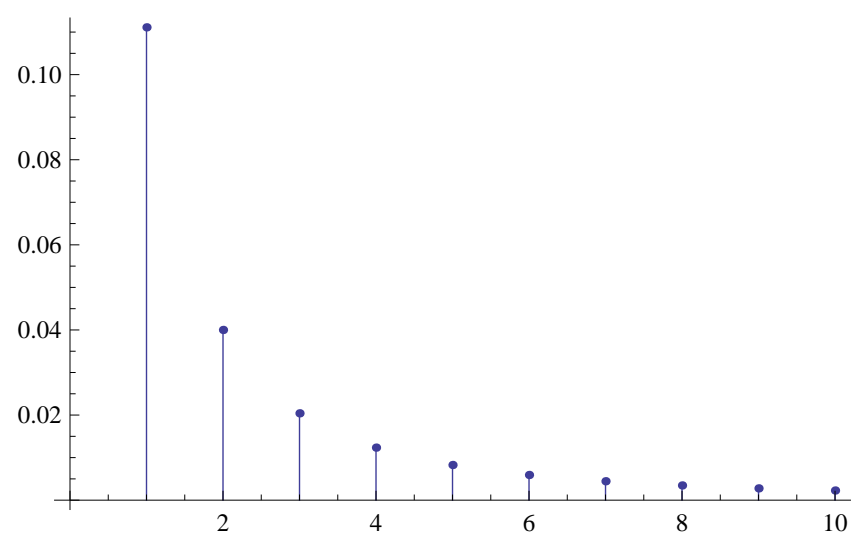

Figure 19: Sequence of $\left(u_{\mathrm{even}}^{(1)}(0)\right)^{2}$ values for $n=1,2, \ldots, 10$ displays relative weights on the brane The $n=0$ mode is about one order bigger than $n=1$ and is not in the figure.

$u_{\text {even }}^{(1)}\left(\alpha_{2}, n, y\right)$ for $n=5,4,3,2,1,-1-2 \alpha_{2}, \ldots,-5-2 \alpha_{2}$, and the eigenfunctions $u_{A S}^{(1)}\left(\alpha_{2}, n, y\right)$ for $n=8,7, \ldots, 1,0,-1-2 \alpha_{2},-2-2 \alpha_{2}, \ldots,-9-2 \alpha_{2}$ are all well-defined tachyonic modes (for larger values of $\epsilon$ the number of tachyons is smaller). Thus, if the dilaton coupling constant happens to be in $\left(\lambda_{0}, \lambda_{0} / 2\right]$, several tachyons would co-exist together with a tower of massive eigenstates of both Maxwell and Kalb-Ramond fields and a Maxwell localized zero-mode.

\section{Final remarks}

In order to determine that massive modes are suppressed as compared with zero-modes, one can evaluate the variation of the effective gauge coupling as a function of the KaluzaKlein photonic masses. Since in the non-relativistic limit the coupling of massive modes with matter on the brane develops a Yukawa type potential, it is natural that massive contributions are strongly attenuated as compared with the Coulomb potential. To show that this quantity is a decreasing function of $m_{A}$ we should evaluate the different coefficients that multiply the four-dimensional action

$$
\sim \int d y\left(u_{m_{A}=0}^{2}(y)+\sum_{n} u_{m_{A}(n)}^{2}(y)\right) \int d^{4} x f_{\mu \nu} f^{\mu \nu}
$$

In order to simplify this computation we shall assume that the coupling with the brane takes place precisely on $4 \mathrm{D}$ ordinary space-time, namely at $y=0$. It is there where the relevant 


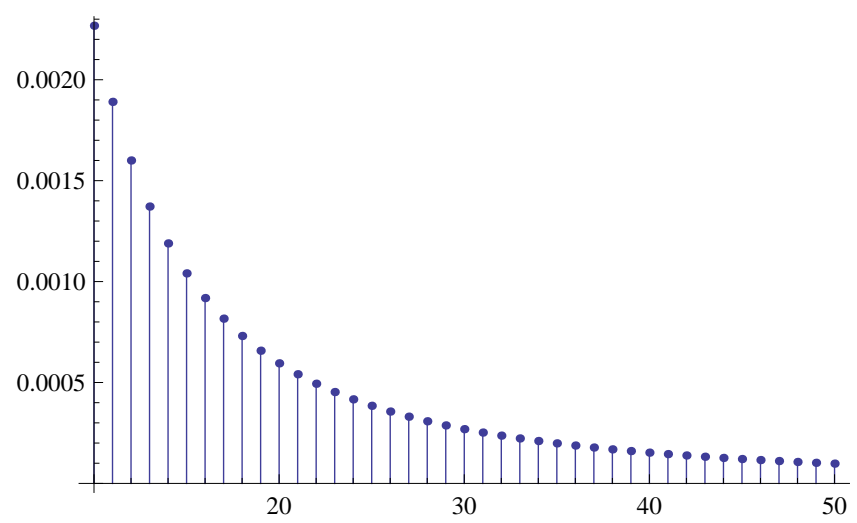

Figure 20: Sequence of $\left(u_{\text {even }}^{(1)}(0)\right)^{2}$ values for $n=10, \ldots, 50$ displays relative weights on the brane $(\alpha=1 / 2)$.

dynamics should be much stronger. Thus, the effective 4D electrostatic potential would read

$$
V(r) \sim q_{1} q_{2}\left(\frac{A^{2}}{r}+\sum_{n} \frac{e^{-m_{A}(n) r}}{r} u_{m_{A}(n)}^{2}(0)\right)
$$

where $q_{1}, q_{2}$ are two test charges separated a distance $r$ in ordinary 3D space and the Kaluza-Klein eigenvalues $m_{A}$ grow with $|n|$ as we have seen. See e.g. Fig. 21 where the $u_{\text {even }}^{(1)}(y)$ modes $n=0,2,50$ are fully displayed. By analytical calculation, for some values of $\alpha(\lambda)$ we can appreciate that at the origin each contribution is indeed rapidly decreasing with mass. See Figs. 19],20, This, together with the negative exponential factor, essentially decouples the massive modes from the physics on the domain wall. Far from the membrane, all massive modes become constants, as much as zero-modes are, and as a consequence 5D phenomenology results completely modified from ordinary 4D electromagnetism. The same analysis can be performed for the Kalb-Ramond modes with the same result. See e.g. Refs. [1, 17] for the study of this issue in the case of gravity.

\section{CONCLUSIONS}

In this paper we have analyzed the existence and localization of Maxwell and KalbRamond propagation modes in a warped extra-dimensional universe. As a result of gauge invariance, the common knowledge is that charged fields must be confined into a fourdimensional brane-world while only neutral fields (under standard model interactions) can be bulk degrees of freedom interacting with the brane. Fermionic and gravitational fields 


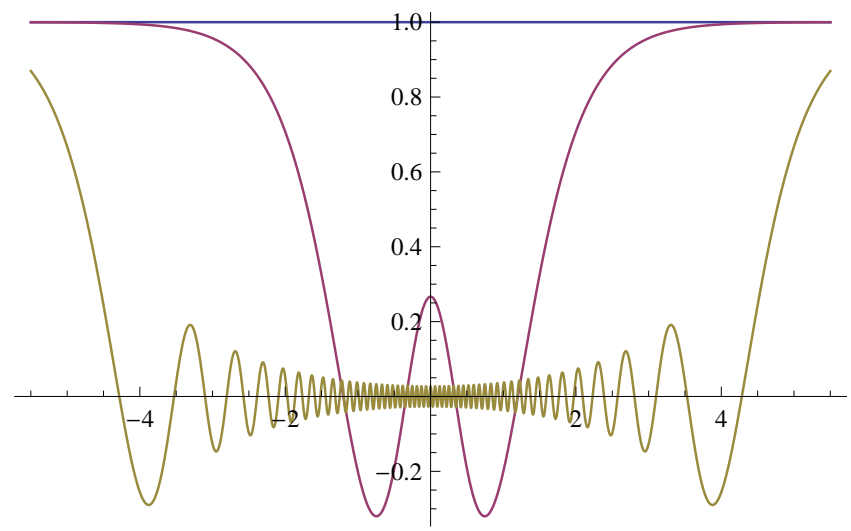

Figure 21: Some $u_{\text {even }}^{(1)}(y)$ modes $n=0,2,50$ (horizontal blue line, two-minima red line, highly oscillating yellow line) exhibit their relative weights. Here $\alpha=3 / 8$.

have been localized in the past rather easily but gauge fields have demanded more effort. As pointed out in [2, 3], the presence of the dilaton happens to be necessary for gauge field modes to be localized in brane-world theories. To analyze this question in a relevant multivacua field theory, we have studied a sine-Gordon like thick membrane that bounces at the (non-compact) extra-coordinate origin. We have focused a five-dimensional metric with two warping functions and two interacting scalar fields. One of them represents the membrane itself and the other represents the dilaton in a field theoretic scenario. The corresponding action includes a potential functional depending on both scalar fields, which is a nontrivial deformation of a sine-Gordon potential and is dynamically consistent with the gravity background. The solution to this action represents a universal framework that interacts with two distinct five-dimensional gauge fields.

To the best of our knowledge, close expressions for all the gauge modes in a 5D spacetime have been obtained here for the first time, particularly for the present combination of field degrees of freedom. Regarding the propagation modes of the Kalb-Ramond field, so far only simple qualitative calculations have been developed in this context. Here, besides performing an analytical approach, we also included the electromagnetic field to treat both of them simultaneously and exhibit their actual differences.

After a detailed analysis of the bulk equations of motion of Kalb-Ramond and Maxwell fields, we have presented the full spectrum of the gauge field problem. The exact variation of the whole set of eigenstates and eigenvalues, with the dilaton coupling constant $\lambda$, was discussed in detail and the exact dependence of vector and tensor gauge modes with the 
extra coordinate was analytically computed in all the cases. As one of our results, we have proven that localization of a Maxwell zero-mode on a smooth thick domain wall embedded in an five-dimensional world is granted for a dilaton coupling constant above $\lambda_{0}$.

Depending on the value of $\lambda$, different phases of the analog potentials related to the equations of motion of Maxwell and Kalb-Ramond fields can arise. If the dilaton coupling constant is on one side or another of determined particular values, the related analog potentials can be dramatically different, allowing for an interpretation of subjacent different physical scenarios (see Sect.VB) . Regarding finite Kaluza-Klein modes, we have analytically found the full spectra of Maxwell and Kalb-Ramond fields and shown that massive modes are strongly suppressed on the brane so that ordinary four-dimensional gauge interactions are not modified in the present set up (see Sect.V C). Among this infinite tower of massive modes, a handful of Kalb-Ramond tachyons could not be excluded unless the dilaton cou-

pling happens to be greater than $\lambda_{0} / 2$. Notably, in this case the theory would lose tachyons but would gain a localized Kalb-Ramond zero-mode. In this way, the present model has been useful to show that a localized zero-mode is not only possible for a 5D electromagnetic field but also, and simultaneously, for a 5D Kalb-Ramond field, depending on just the value of the dilaton coupling constant. As a general conclusion, the above results help showing that the model presented here is an interesting arena to discuss extra-dimensional physics and that our ordinary 4D world seems to be compatible with a higher dimensional universe, apparently of a stringy brane nature.

\section{Acknowledgments}

H.R.C. acknowledges FUNCAP for financial support and F.A. Schaposnik for useful comments.

[1] L. Randall and R. Sundrum, Phys. Rev. Lett. 83, 4690 (1999).

[2] A. Kehagias and K. Tamvakis, Phys. Lett. B 504, 38 (2001).

[3] D. Youm, Nucl. Phys. B 589, 315 (2000).

[4] A. Pomarol, Phys. Lett. B 486, 153 (2000). 
[5] V. E. Lopatin and M. A. Vasiliev, Mod. Phys. Lett. A 3, 257 (1988); C. Germani and A. Kehagias, Nucl. Phys. B725, 15 (2005).

[6] J.Polchinski, String Theory, vols. 1 \& 2, (Cambridge University Press, Cambridge, England, 1998).

[7] M. Kalb and P. Ramond, Phys. Rev. D9 2273 (1974).

[8] S.Kar, S. SenGupta and S. Sur, Phys. Rev. D67 044005 (2003), P. Jain, S. Panda, S. Sarala Phys. Rev. D66 085007(2002); Y. Itin , F.W. Hehl, Phys. Rev. D68 127701 (2003).

[9] T. Gherghetta, A. Pomarol, Nucl. Phys. B 586 (2000) 141; B. Bajc, G. Gabadadze, Phys. Lett. B 474 (2000) 282; Yu-Xiao Liu, Li-Da Zhang, Li-Jie Zhang, Yi-Shi Duan, Phys. Rev. D 78065025 (2008); C. A. Almeida, R. Casana Sifuentes, M. M. Ferreira, A. R. Gomes, Phys. Rev. D 79125022 (2009).

[10] G. Dvali, M. Shifman, Phys. Lett. B 396 (1997) 64; B 407 (1997) 452 Erratum.

[11] M. O. Tahim, W. T. Cruz, and C. A. Almeida, Phys. Rev. D 79085022 (2009).

[12] D. Youm, Phys. Rev. D64, 127501 (2001).

[13] M. Cvetic, S. Griffies and S. Rey, Nucl. Phys. B 381, 301 (1992).

[14] K. Skenderis, P.K. Townsend, Phys. Lett. B 468 (1999) 46.

[15] A. Kehagias, Phys. Lett. B469 (1999) 123.

[16] M. Gremm, Phys. Lett. B 478, 434 (2000).

[17] C. Csaki, J. Erlich, T. J. Hollowood, and Y. Shirman, Nucl. Phys. B581, 309 (2000). C. Csaki, J. Erlich, T. J. Hollowood, Phys. Rev. Lett. 84, 5932 (2000).

[18] Saurya Das, Anindya Dey, Soumitra SenGupta, Class. Quant. Grav. 23, L67 (2006).

[19] B. Mukhopadhyaya, S. Sen, S. SenGupta, Phys. Rev. Lett. 89 (2002) 121101; Erratum-ibid. 89 (2002) 259902.

[20] W. D. Goldberger, M. B. Wise, Phys. Rev. Lett. 83 (1999) 4922; J. Garriga, O. Pujolas and T. Tanaka, Nucl. Phys. B605 (2001) 192.

[21] C. Csaki, M. Graesser, L. Randall and J. Terning, Phys. Rev. D 62 (2000) 045015;C. Csaki, M. Graesser and G. Kribs, Phys. Rev. D 63 (2001) 065002; O. DeWolfe, D. Freedman, S.S. Gubser and A. Karch, Phys. Rev. D62 (2000) 046008.

[22] K. Sfetsos and A.A. Tseytlin, Phys. Rev. D 49, 2933 (1994); B. Kleihaus, J. Kunz and K. Myklevoll, Phys. Lett. B 605, 151 (2005).

[23] P. Mayr and S. Stieberger, Nucl. Phys. B 412, 502 (1994). 
[24] H. Taşeli, J. Math. Chem. 34, 243 (2003). 